\title{
tic\&société
}

Vol. 7, $\mathrm{N}^{\circ} 1 \mid$ 1 er semestre 2013

Formes et enjeux de la collaboration numérique

\section{Travailler à haute voix sur Twitter}

Quand la collaboration informelle emprunte un réseau public

\section{Claudine Bonneau}

\section{(2) OpenEdition}

Journals

Édition électronique

URL : http://journals.openedition.org/ticetsociete/1330

DOI : 10.4000/ticetsociete.1330

\section{Éditeur}

Association ARTIC

\section{Référence électronique}

Claudine Bonneau, «Travailler à haute voix sur Twitter », tic\&société [En ligne], Vol. 7, No 1 | 1er semestre 2013, mis en ligne le 05 juin 2013, consulté le 20 avril 2019. URL : http:// journals.openedition.org/ticetsociete/1330 ; DOI : 10.4000/ticetsociete.1330

Ce document a été généré automatiquement le 20 avril 2019

Licence Creative Commons 


\title{
Travailler à haute voix sur Twitter
}

Quand la collaboration informelle emprunte un réseau public

\author{
Claudine Bonneau
}

\section{Introduction : les usages de Twitter en contexte organisationnel}

1 Les usages expressifs d'Internet et les pratiques d'auto-dévoilement en ligne se sont accrus avec l'arrivée des médias sociaux (Proulx, Millette et Heaton, 2012). Par leur design, ces derniers encouragent les usagers à diffuser leurs intentions, idées et opinions dans un espace public plus large.

2 Les pratiques de microblogage réfèrent plus spécifiquement à la publication de brefs messages textuels par l'entremise d'outils numériques tels que Twitter et Tumblr ainsi que les mises à jour de «statuts » sur les sites de réseaux sociaux tels que Facebook et LinkedIn. Dans cet article, nous nous intéressons plus particulièrement à l'outil de microblogage Twitter, utilisé par 140 millions d'internautes (Twitter, 2012) tout à la fois à des fins personnelles et professionnelles (Huberman, Romero et $\mathrm{Wu}, 2009$ ).

3 Parmi les usagers de cette plateforme de microblogage, on retrouve des travailleurs qui l'utilisent pour diffuser des connaissances tacites ou formalisées, mais aussi des hypothèses et pistes de réflexion qui jalonnent leurs pratiques professionnelles. Cette plateforme leur permet de communiquer en temps réel avec un public excédant les frontières de leur organisation de travail.

4 Les conversations portant sur la conduite du travail empruntent ce canal public qui n'est pas nécessairement reconnu ni contrôlé par les employeurs, à travers des initiatives informelles des travailleurs, plutôt qu'en réponse à des procédures prescrites par l'organisation.

5 Bien que plusieurs recherches en sociologie du travail se soient penchées sur l'étude des modes alternatifs de présence au travail (Bødker et Christiansen, 2006), c'est-à-dire hors des lieux ou des temps contractuels (Crague, 2003 ; Ladner, 2008), ainsi que sur les 
nouvelles formes d'interactions et de collaboration rendues possibles par les outils de communication en temps réel, telles que les requêtes informationnelles et les questions de clarification lancées aux collègues via les messageries instantanées (Licoppe, Cudicio et Proulx, 2011), les usages des médias sociaux au sein des milieux de travail ont été peu étudiés par les chercheurs s'intéressant à la communication et à l'utilisation des technologies d'information et de communication en organisation. Lors d'un récent appel à contribution, Steinfield et al. (2012) déplorent que les chercheurs en communication ne s'intéressent, en règle générale, qu'aux utilisations marketing ou publicitaires et négligent de traiter de l'influence des médias sociaux sur les processus de communication organisationnelle. Une recension de la littérature ${ }^{1}$ permet de justifier et de préciser cette affirmation, en montrant une sureprésentation des études portant sur l'utilisation des médias sociaux à des fins de marketing (Bonneau, 2012 ; Diffley et al., 2011 ; LorenzoRomero, Constantinides et Alarcón-del-Amo, 2011), de relations publiques (Croft, 2007 ; Diga et Kelleher, 2009) ou encore de relations avec la clientèle, l'actionnariat ou les fournisseurs (Rybalko et Seltzer, 2010).

6 Par ailleurs, on note que les revues phares telles qu'Organization Science et Organization Studies abordent les problématiques du changement organisationnel depuis des perspectives relativement éloignées de celle de l'utilisation des médias sociaux au travail, en traitant notamment de la gestion des différentes technologies en contexte de collaboration (Bailey, Leonardi et Chong, 2009), des formes de collaboration dans les communautés en ligne (Faraj et Johnson, 2010) ou encore de l'impact des interruptions causées par les technologies de communication sur les capacités de concentration des employés (Wajcman et Rose, 2011), pour ne citer que quelques exemples.

7 En fait, les rares études s'intéressant au potentiel des médias sociaux pour le travail collaboratif en contexte organisationnel se trouvent au sein de la littérature produite par les chercheurs du domaine du Computer Supported Collaborative Work (CSCW) et du HumanComputer Interaction (HCI). Toutefois, ces derniers se sont surtout attardés à des versions " corporatives " de ces plateformes qui sont destinées à un public interne (Rooksby et Sommerville, 2011). En somme, toute la mouvance à laquelle réfère l'expression " Entreprise 2.0 ( (McAfee, 2006) concerne avant tout des plateformes collaboratives telles que des blogues internes (Huh et al., 2007 ; Jackson, Yates et Orlikowski, 2007 ; Liao et al., 2011 ; Yardi et al., 2009), des microblogues privés (DiMicco et al., 2008 ; Zhang et al., 2010) ou des wikis d'entreprise (Hasan et Pfaff, 2006 ; Stocker et al., 2012). En effet, malgré une volonté de transformer l'intranet d'entreprise en une plateforme plus organique, collaborative et gérée par l'utilisateur, la gestion des connaissances et de la collaboration s'effectuent généralement par l'entremise de plateformes collaboratives (groupware) implantées officiellement par l'organisation, suivant une dynamique descendante où l'employeur dicte les principes et les façons de faire à ses employés. En ce sens, les médias sociaux publics sont rarement considérés comme étant des plateformes collaboratives appropriées en contexte organisationnel, si bien que près du tiers (32\%) des entreprises canadiennes les jugent nuisibles pour la productivité de leurs employés et interdisent complètement leur utilisation au bureau, même pour des raisons d'affaires (Robert Half Technologies, 2011).

8 Néanmoins, les internautes qui utilisent déjà les plateformes publiques de microblogage dans leur vie personnelle ont de plus en plus le réflexe de les intégrer à leurs activités professionnelles. En effet, même dans les entreprises qui mettent des outils de collaboration « officiels » à la disposition de leurs employés, près du tiers d'entre eux 
utilisent les outils non-officiels (Tay, 2011). Zhao et Rosson (2009) ont réalisé une étude exploratoire afin d'examiner les impacts de Twitter sur la communication informelle au travail. Bien qu'ils aient noté que certaines initiatives de collaboration pouvaient naître par l'entremise de cette plateforme de microblogage, ces chercheurs se sont concentrés exclusivement sur les interactions avec les collègues de la même organisation dans un contexte défini et structuré (un objectif connu et des rôles organisationnels identifiés).

Ehrlich et Shami (2010) ont pourtant montré que dans une organisation où cohabitent deux plateformes de microblogage, l'une privée, l'autre publique, les types de contenus diffusés par les employés qui les utilisent sont différentes. Par exemple, les usagers ont tendance à chercher des informations ponctuelles sur l'outil interne et font circuler de l'information ou fournissent des réponses sur le réseau public. Comme le souligne Papacharissi (2009), les architectures et contextes d'implantation propres à chaque plateforme créent des affordances communicationnelles différentes. La cartographie du Web 2.0 proposée par Cardon (2008) souligne également les spécificités en matière de visibilité des profils et des interactions des usagers en fonction du type d'outil. Ainsi, les médias sociaux publics mobilisés au travail appartiendraient davantage à la catégorie du «Phare » où la visibilité est forte autour de thèmes précis (on retrouve là autant LinkedIn que Twitter) et où l'on sollicite davantage les liens faibles (Granovetter, 1973) puisque les interactions qui ont cours sont centrées autour du «faire».

Dans ce contexte, il nous apparaît important d'examiner plus en détail ce que les travailleurs racontent ouvertement sur Twitter à propos du travail qu'ils sont en train de réaliser. Ces usages peuvent impliquer des interactions avec des personnes à l'extérieur de l'organisation que l'usager ne connaît pas nécessairement.

11 Dans cet article, on trouvera d'abord une brève mise en contexte décrivant les particularités fonctionnelles et communicationnelles de Twitter. Puis, nous examinerons des exemples de messages illustrant chaque type d'usage de cette plateforme de microblogage lié à la conduite du travail et à la collaboration informelle.

\section{Spécificités matérielles et communicationnelles de Twitter}

12 Lancé en juillet 2006, la plateforme de microblogage répond à des besoins potentiellement différents de ceux déjà comblés par les autres outils de communication médiatisés par ordinateur mis à la disposition des travailleurs, tels que le courriel, la messagerie instantanée et les blogues. Dans les prochaines sous-sections, nous mettrons l'emphase sur les propriétés matérielles et les fonctions communicationnelles qui distinguent Twitter des autres plateformes de communication afin de pouvoir montrer comment elles facilitent ou contraignent les usages qui nous intéressent.

\subsection{Concision des messages}

Les usagers s'étant préalablement inscrits à cette plateforme disposent d'un compte à partir duquel ils peuvent publier des messages tweet, c'est-à-dire des mises à jour textuelles (composées de 140 caractères maximum) auxquelles peuvent être intégrés des hyperliens menant à des sites web, des images, des vidéos, etc. La concision des messages est l'une des particularités qui distingue cette plateforme des blogues, en réduisant le 
coût cognitif (Bonneau et Harvey, 2006) associé aux efforts nécessaires pour publier. D'ailleurs, la fréquence de publication est généralement plus régulière sur Twitter que sur un blogue (Sakaki, Okazaki et Matsuo, 2010) parce que la publication d'un tweet de 140 caractères est beaucoup plus rapide et moins exigeante pour l'usager que celle d'un billet de blogue (Zhao et Rosson, 2009).

La concision des tweets module également la façon dont ils sont lus, tout en facilitant la consultation d'une grande quantité de mises à jour par les lecteurs. En effet, le format des publications permet aux lecteurs de parcourir rapidement les mises à jour des usagers auxquels ils sont abonnés et de déterminer celles qui les intéressent, comme s'ils survolaient les grands titres d'un quotidien.

\subsection{Asymétrie des abonnements et diffusion publique par défaut}

15 Le caractère asymétrique des abonnements distingue cette plateforme de microblogage des médias sociaux où les " liens d'amitié " sont obligatoirement réciproques. En effet, l'usager de Twitter peut s'abonner aux comptes d'autres usagers sans qu'il y ait réciprocité. Le fait d'être abonné à un compte permet de suivre ses mises à jour dans son fil d'actualités. Les usagers disposant d'un grand nombre d'abonnés à leur compte se retrouvent ainsi dans une dynamique de diffusion à une large audience (broadcast), composée à la fois de connaissances et d'inconnus.

Lors de l'ouverture d'un compte, il est public "par défaut », de sorte que quiconque peut s'y abonner sans avoir à obtenir une permission. Cette «politique de visibilité » (Cardon, 2008) suggérée par Twitterfacilite la découverte de nouveaux comptes par sérendipité. Ainsi, les conversations qui empruntent ce canal sont visibles par tous, à condition que les usagers n'aient pas configuré leur compte de manière à ce qu'il soit privé.

17 L'asymétrie et le caractère public des abonnements sur la plateforme de microblogage étudiée contribuent à la rendre moins intrusive que d'autres moyens de communication numériques «directs» tels que le courriel ou la messagerie instantanée puisque les messages sont destinés à une audience plus ou moins large, les destinataires ressentent ainsi moins de pression pour y répondre (Zhao et Rosson, 2009). Du côté de la publication, cela permet aux usagers de l'utiliser de manière informelle pour exprimer des idées ou lancer des questionnements qu'ils ne sont pas en mesure d'adresser à quelqu'un en particulier (bien que cela soit possible, comme nous le verrons ultérieurement lorsque nous aborderons les " publications dirigées ».)

\subsection{Mobilité et diversité des méthodes d'accès}

Twitter est une application en ligne à laquelle il est possible d'accéder par le biais d'un navigateur web, d'un logiciel client installé sur l'ordinateur, d'une application mobile et d'un nombre toujours grandissant de composantes intégrées au sein de plateformes de tout acabit. Que l'usager soit devant son ordinateur ou qu'il utilise son téléphone mobile en déplacement, il dispose toujours d'un moyen simple d'accéder à son compte.

19 Cette diversité des méthodes d'accès, combinée au format concis des messages font que Twitter est l'un des outils les plus simples d'accès et d'utilisation à partir d'un appareil mobile, si bien que plus de la moitié des abonnés accèdent à cette plateforme à partir de leur mobile (Pew Internet \& American Life Project, 2011). D'ailleurs, l'imposition de la 
limite de 140 caractères avait initialement été pensée en fonction des messages SMS qui sont limités à 160 caractères (20 caractères sont conservés par Twitter pour ajouter le nom d'utilisateur). La liberté et la mobilité qui caractérisent le service de messagerie texte (SMS) propre à la téléphonie mobile se retrouvent au cœur des fonctionnalités de cette plateforme de microblogage. Celle-ci peut ainsi suivre l'usager dans toutes ses activités personnelles et professionnelles et être utilisé en situation multitâche, par exemple, lorsqu'un usager publie des messages pendant une réunion à laquelle il assiste. Même si l'employeur restreint l'accès à la plateforme à partir des ordinateurs utilisés par ses employés, ces derniers peuvent toujours utiliser leurs téléphones mobiles personnels pour y accéder discrètement sur leurs lieux de travail.

\subsection{Le caractère éphémère de la diffusion en temps réel}

20 L'immédiateté et l'instantanéité caractérisent la diffusion et l'accès à l'information en temps réel sur la plateforme étudiée. En effet, la concision des tweets et l'accès en mobilité incitent les usagers à publier leurs messages en temps réel de façon spontanée, au fur et à mesure que survient un événement ou qu'ils prennent connaissance d'un contenu digne de mention, plutôt que d'attendre d'avoir le temps de publier une réflexion plus élaborée après coup. Les usagers y partagent de l'information au moment où elle devient disponible, de sorte que la plupart des événements (sportifs, politiques, culturels), des catastrophes (tremblements de terre, ouragans, incendies) ou des nouvelles (actualités de dernière heure, scoops) sont rapidement annoncées souvent avant même d'être diffusés par les médias traditionnels (Sakaki, Okazaki et Matsuo, 2010).

Ces publications en temps réel se succèdent à un rythme effréné sur la page d'accueil de l'usager qui affiche les tweets des comptes auxquels il est abonné de façon antéchronologique, créant un flux d'information en continu. Même si techniquement, les tweets persistent au-delà de leur publication, de sorte qu'il est possible d'y accéder de façon asynchrone en retournant en arrière ou en lançant une recherche, reste que la fréquence de mise à jour des tweets leur confère un caractère éphémère. En effet, les tweets plus anciens sont immédiatement remplacés par les nouveaux et finissent par être relégués vers des espaces plus rarement consultés. Ainsi, les usagers de cette plateforme lisent généralement les tweets au moment de leur publication (ou peu de temps après) et l'utilisent comme plateforme de communication synchrone (ou presque).

\subsection{Publications dirigées, rediffusion et mots-clics}

En plus de la possibilité de diffuser des informations à une large audience, la plateforme est également utilisée pour adresser des messages à des comptes ciblés. Nous utilisons le terme de "publication dirigée " pour désigner ce type de communication directe, qui n'est pas un message privé, mais bien un tweet «ouvert " (Huberman, Romero et Wu, 2009). En effet, l'utilisation du caractère "arobas" suivi du nom d'usager (ex.: @clobonneau) permet d'adresser le tweet à un usager (ou plusieurs), qui le verront ainsi listé dans leur rubrique "Connecter ». L'utilisation de ce "marqueur de destination ${ }^{2}$ est une innovation des usagers qui en avaient besoin pour attirer l'attention d'un autre usager sur leur tweet, que ce soit pour poser une question, cibler l'envoi d'un commentaire ou participer à une discussion. En effet, les concepteurs n'avaient pas prévu 
initialement que leur plateforme serait utilisée pour mener des conversations (Honeycutt et Herring, 2009).

Ce marqueur, précédé de l'acronyme RT (pour retweet), est également utilisé pour rediffuser le message d'un autre usager à ses abonnés, de manière à citer son auteur. En plus d'être une forme de reconnaissance, la rediffusion permet de donner à ces usagers des nouvelles audiences (Boyd, Golder et Lotan, 2010). En effet, les pratiques de rediffusion rendent plus visibles les liens entre usagers en permettant de savoir « qui est abonné à qui ».

Finalement, les usagers peuvent intégrer dans leurs messages des mots-clics (hashtag) ${ }^{3}$ composés du symbole dièse suivi d'un mot-clé (ex. : \#boulot) afin de référencer le contenu de leur message. Les mots-clics permettent une mise en contexte et facilitent ensuite la recherche thématique. Un hyperlien est automatiquement associé au mot-clic pour le rendre cliquable, permettant ainsi d'accéder à la liste de tous les messages qui l'incluent. Outre ses capacités de classification, le mot-clic peut donner lieu à une forme de narration collective particulière, lors de laquelle plusieurs usagers (n'étant pas nécessairement abonnés entre eux) tiennent une conversation ouverte autour d'un sujet. Cette fonction est particulièrement utile dans le cas de séances de live tweet lors desquelles les usagers publient des messages concernant un événement en direct (ex. : émission de télévision en ondes, actualité brûlante, etc.).

Les fonctions que nous venons de décrire sont mobilisées de façon particulière par certains travailleurs pour raconter leur vie professionnelle et collaborer de façon informelle. Dans la prochaine section, nous allons examiner quelques exemples de messages publiés en fonction de différents types d'usages qui en sont faits au travail.

\section{Du récit à la collaboration : caractériser les usages informels au travail}

Des classifications ont déjà été proposées pour inventorier les catégories de contenu publiées sur la plateforme étudiée (Dann, 2010) ainsi que les motivations des usagers (Domenget, 2012 ; Huberman, Romero et $\mathrm{Wu}, 2009$ ). Pour notre part, nous excluons d'emblée les messages visant à faire la promotion et la valorisation d'un travail " fini " (ex. : le journaliste qui diffuse le lien vers son plus récent reportage ou le chercheur qui présente ses résultats de recherche). En effet, de tels usages rejoignent ce qui a déjà été dit sur le potentiel de cette plateforme à des fins de promotion, de marketing ou de relations publiques. Nous ne prendrons pas en compte les messages destinés à diffuser des connaissances déjà formalisées (ex.: partager un article pertinent au domaine professionnel), puisque de telles activités de veille de contenu et de curation - rappelons que la curation de contenu repose sur trois composantes : la sélection, l'éditorialisation et le partage - ont déjà été amplement abordées dans la littérature portant sur les médias sociaux. Les différents types d'usages que nous présentons au fil des prochaines soussections sont illustrés à l'aide d'exemples de messages qui prennent plutôt la forme de récits, de publications ou de conversations en lien avec le travail "en train de se faire" et lors de la mise en œuvre de collaborations informelles.

Ces usages peuvent être étudiés notamment à travers les traces laissées par les usagers qui ont décidé de les rendre accessibles publiquement. D’un point de vue méthodologique, notre démarche qualitative s'inspire de l'ethnographie en ligne (Hine, 2000). Dans cet 
article, nous utilisons un corpus composé d'une cinquantaine de messages publics qui ont été recueillis lors de la première phase d'une étude exploratoire auprès d'un échantillon non-représentatif composé de quinze usagers de Twitter, dont trois femmes et douze hommes résidant au Canada, aux États-Unis et occupant des emplois dans les secteurs des technologies de l'information, du journalisme, de la recherche académique et de l'enseignement supérieur. L'échantillon a été construit de manière non probabiliste puisque la recherche des messages a été orientée en fonction des objectifs de la recherche et de l'accessibilité des profils (publics ${ }^{4}$ ) et leur langue (français ou anglais).

L'analyse de ces messages repose sur une codification thématique manuelle de manière ouverte et inductive (Miles et Huberman, 1994). La grille d'analyse a été conçue à partir des thèmes préconisés en fonction de notre question de recherche. Chaque concept associé à l'un de ces thèmes est identifié par un marqueur (mot-clé). Ces marqueurs ont ensuite été utilisés pour coder et organiser le corpus de messages. Nous avons gardé la grille d'analyse ouverte afin de pouvoir l'enrichir au fil de la cueillette et de l'analyse des données. En retravaillant le corpus, de nouveaux thèmes et concepts sont apparus et ont ainsi pu être ajoutés à la grille.

\subsection{Raconter le travail en train de se faire : une invitation à la conversation}

29 Alors qu'on demande de plus en plus aux travailleurs de produire des rapports et compterendu de leur travail afin de le rendre plus visible à leurs collègues et supérieurs, certains trouvent d'autres façons de rendre compte de leurs pratiques qui sinon, risqueraient de demeurer invisibles. Dave Winer ${ }^{5}(2009)$ a été l'un des premiers à souligner le potentiel des outils de publication en ligne pour « raconter le travail ». Même s'il ciblait d'abord le travail des journalistes, ce type d'usage est repris dans d'autres domaines professionnels. Cette pratique a également été désignée par l'expression «travailler à haute voix » ( working out loud) (Williams, 2010), une formulation dérivée de l'expression "penser à haute voix " (thinking out loud) qui illustre bien la volonté de rendre son travail visible au fur et à mesure qu'il prend forme au lieu de simplement l'énoncer au moment de le planifier ou en rendre compte après qu'il ait été complété.

La possibilité de diffuser rapidement de courts messages en temps réel fait de Twitter une plateforme toute désignée pour «travailler à haute voix ». En effet, certains travailleurs utilisent celle-ci pour raconter leur journée de travail, donner à voir leur activité professionnelle, voire la mettre en scène. Par exemple, une programmeure annonce à ses abonnés qu'elle vient de compléter un jalon important de son projet de développement logiciel, ou encore, un entrepreneur indique qu'il vient de terminer une réunion productive avec un partenaire potentiel. Ces récits sont parfois très précis, surtout lorsque les usagers ont recours à des liens pour compléter leur message, par exemple, en insérant un lien vers une page renfermant le code de programmation de la composante du logiciel ou en publiant la carte géographique montrant le lieu de la réunion. 


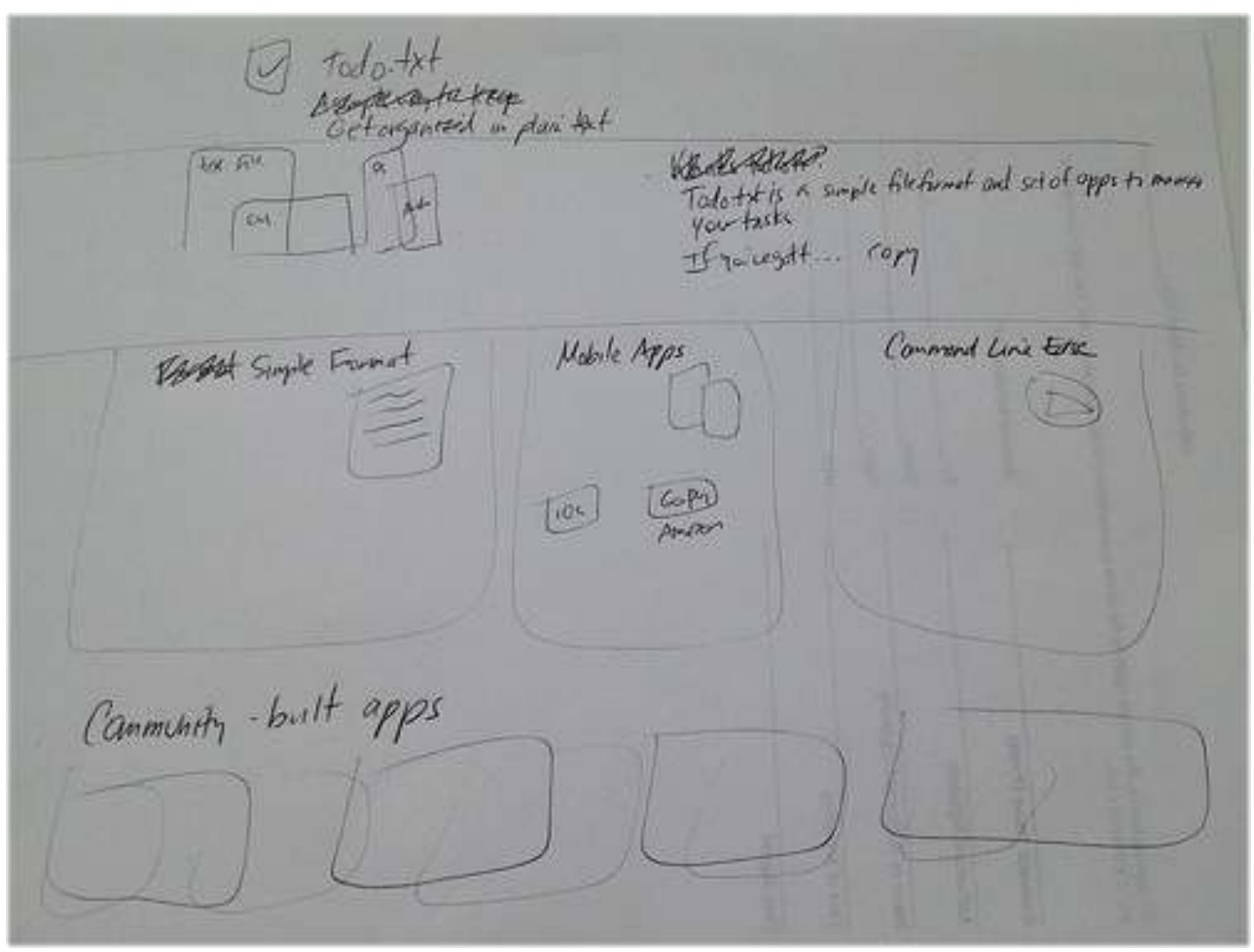

31 Dans l'exemple illustré (cf. figure 1), la programmeure décrit la méthode qu'elle emploie au travail en publiant une photo de la maquette qu'elle vient de griffonner. Elle montre à ses abonnés que bien qu'elle soit une fervente utilisatrice de la tablette iPad et de ses applications de dessin sophistiquées,

elle dessine régulièrement sur une feuille de papier. Après avoir publié ce message, elle recevra de nombreuses réponses d'autres usagers qui lui avouent procéder de la même façon ou qui lui parlent de leurs méthodes respectives.

Ces récits, qui sont destinés à tous (et à personne en particulier), constituent souvent le point de départ de conversations, parce que d'autres usagers se sentent concernés et souhaitent ajouter leur grain de sel. Alors que Honeycutt et Herring (2009) considéraient que les conversations sur cette plateforme de microblogage pouvaient être repérées uniquement à travers des messages renfermant un marqueur de destination (ex.: @nomusager), nous avons plutôt vu des formes de conversations collectives émerger à partir d'un récit qui ne s'adressait à personne en particulier (et qui n'incluait donc aucun marqueur). Ces «tranches de vie professionnelles » sont parfois destinées à exprimer des émotions et évacuer des tensions auprès de personnes n'étant pas directement impliquées, comme dans l'exemple illustré (cf. figure 2) où une designer Web prend le temps de partager le déroulement de sa journée de travail. 
Figure 2 : Évacuer des tensions auprès d'une autre audience

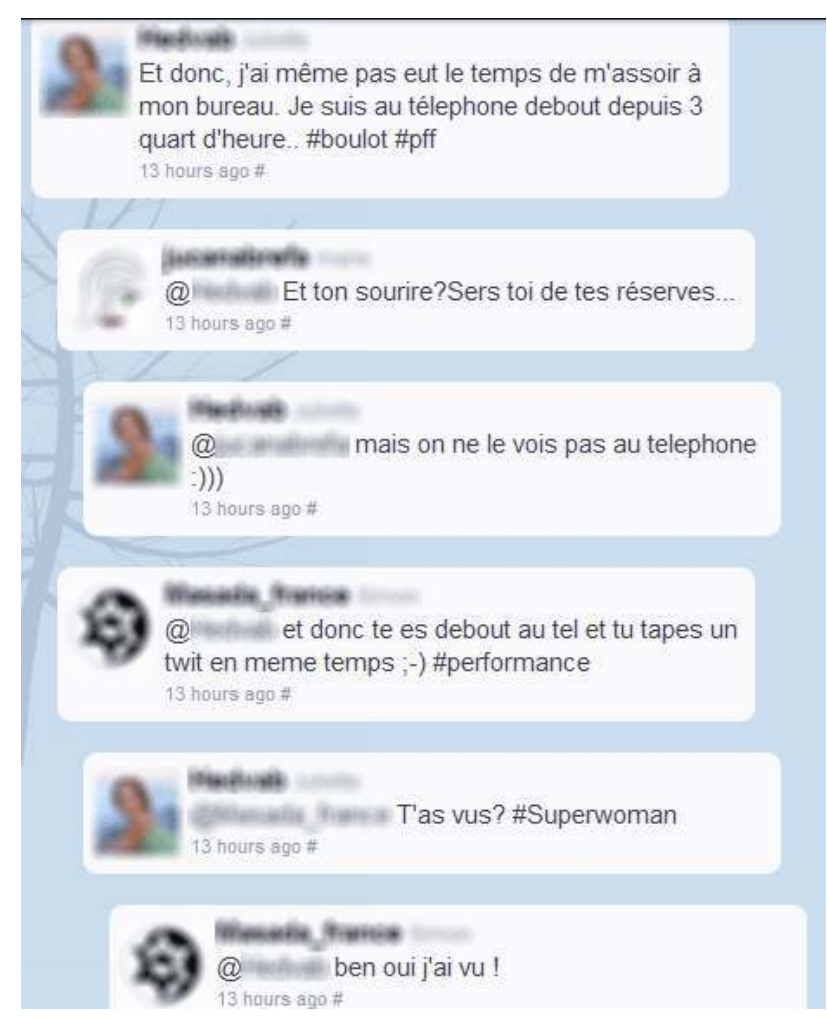

Ces courts récits servent en quelque sorte de points d'ancrage sur lesquels s'appuient des interactions avec d'autres usagers. Le caractère "public par défaut» des comptes de microblogage et l'asymétrie des abonnements permettent d'ouvrir la conversation à d'autres usagers que les collègues de travail. Les récits portant sur le travail constituent l'un des moyens pour les usagers de développer un terrain commun et un sentiment de proximité avec des usagers avec qui ils collaboreront peut-être éventuellement.

\subsection{Partager des traces du travail en train de se faire : une invitation à la collaboration}

Certains travailleurs ne se contentent pas de raconter ou d'illustrer leurs activités professionnelles : ils donnent directement accès à une forme "en construction" du travail, qu'il s'agisse d'un fichier éditable, d'une production incomplète ou perfectible ou de toute autre trace de leur réflexion en cours. Par exemple, un directeur marketing diffuse un lien vers la présentation Powerpoint qu'il est en train de créer, mais qui n'est pas terminée. Dans l'exemple illustré (figure 3), un professeur d'université inclut dans son message un lien vers un document Google Docs dans lequel il recueille ses idées pour une activité pédagogique en développement. De par son support, le document est ouvert aux contributions de tous. Il obtiendra effectivement des suggestions de la part de certains de ses abonnés qui iront directement contribuer à son document afin d'affiner son idée de départ d'une manière collaborative. 


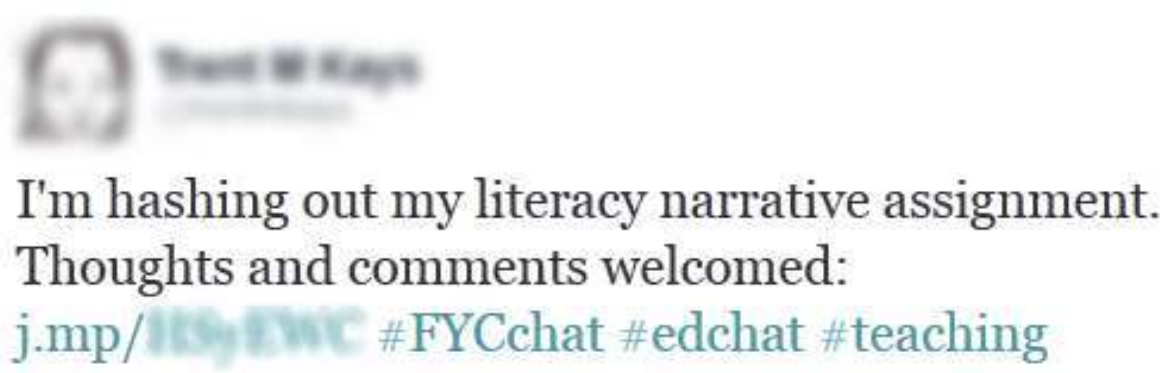

Cette forme de «travail ouvert » repose sur l'idée que d'une part, il est plus productif et efficace de rendre son travail visible et disponible à tous plutôt que de le cacher et d'autre part, que cela doit s'effectuer au moment même où le travail prend forme plutôt qu' $a$ priori ou a posteriori, quitte à en présenter des formes imparfaites et incomplètes de type work in progress. Malgré leur forme très courte, ces messages constituent une occasion pour le travailleur de prendre du recul face à l'activité de travail en cours, mais surtout, de rendre ce processus réflexif plus visible et social en l'ouvrant à une audience afin d'obtenir de la rétroaction.

\subsection{De la diffusion d'une intention à la coordination de l'action}

Certains travailleurs utilisent la plateforme de microblogage pour diffuser leurs intentions avec un public excédant les frontières de l'organisation qui les embauche. Ici, au lieu de raconter, montrer ou donner accès au travail en train de se faire, on partage des réflexions à propos d'un travail qui pourrait être entrepris. Bref, on évoque une opportunité afin de lui donner de la visibilité, d'en tester la faisabilité ou d'évaluer l'intérêt qu'elle suscite. Le fait de signaler une intention constitue donc une première étape destinée à ressembler des gens susceptibles d'être intéressés, en la faisant circuler à travers l'audience du travailleur et de celle des abonnés qui la rediffuseront à leur tour. $\mathrm{Au}$ fil de ce parcours, elle génère un ensemble de réponses et de commentaires prenant la forme d'une séance de remue-méninges distribuée. Ici, le microblogage permet de réfléchir ensemble, de repérer de potentiels collaborateurs et de commencer à coordonner l'action. Ce type d'usage correspond à ce que von Kaufmann (2009) avait désigné par l'expression intention broadcasting, qui réfère au partage et à la coordination des intentions médiées par (ou assistées par) les technologies de l'information.

Qu'il s'agisse de concevoir un logiciel, d'organiser un événement ou de mettre sur pied une association, le message invite à un futur possible. On ne sait pas encore comment l'intention sera opérationnalisée, mais on sait qu'on ne pourrait y arriver seul, d'où le recours au microblogage pour repérer d'autres usagers partageant le même intérêt. Dans l'exemple illustré (cf. figure 4), le directeur des services technologiques d'une école secondaire évoque l'idée de créer un programme pédagogique qui s'inspirerait d'un ouvrage récemment publié. En diffusant cette intention, il en profite pour ajouter le marqueur de destination de l'auteur pour lui demander son avis. Ce dernier répondra d'ailleurs immédiatement à son message en l'invitant à collaborer à un syllabus qu'il a commencé à concevoir en lien avec son bouquin. 


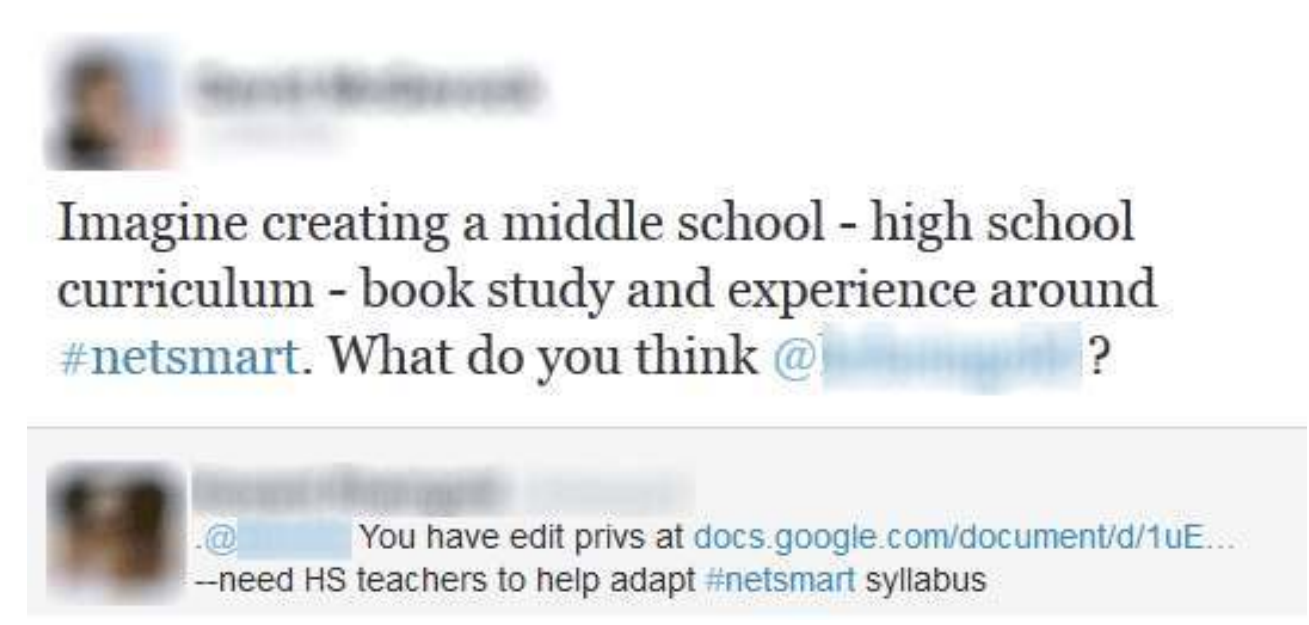

\subsection{Questionner la foule: un bénéfice personnel partagé}

Des recherches ont déjà montré que les plateformes de microblogage sont souvent utilisées pour obtenir de l'aide et des réponses en formulant des demandes « à la foule », à la manière du crowdsourcing (externalisation vers la foule) (Domenget, 2012 ; Ehrlich et Shami, 2010). Les capacités de diffusion de masse propres à Twitter et le jeu du relai ( retweet) font en sorte d'orienter la diffusion d'une question ou d'une demande d'aide à des usagers en mesure d'y répondre, sans avoir besoin de savoir " qui sait quoi », ou " qui sait qui sait quoi » (Nardi, Whittaker et Schwarz, 2000). Ici, même si l'usager n'a pas développé de liens avec des communautés épistémiques formés d'experts partageant des connaissances à propos d'un domaine, il mobilise son réseau de "liens faibles" (Granovetter, 1973) pour trouver des ressources en-dehors de leur milieu de travail. Une étude a d'ailleurs montré que $42 \%$ des usagers de Twitter sont susceptibles de répondre à une question spécifique posée par un étranger sur le réseau et que $44 \%$ des réponses arrivaient en deçà d'un délai de trente minutes (Nichols et Kang, 2012). Une designer Web (cf. figure 5) demande de l'aide pour réaliser un type d'effet visuel, et reçoit dans l'heure suivante des réponses de la part d'autres designers ne travaillant pas au sein de la même organisation. 


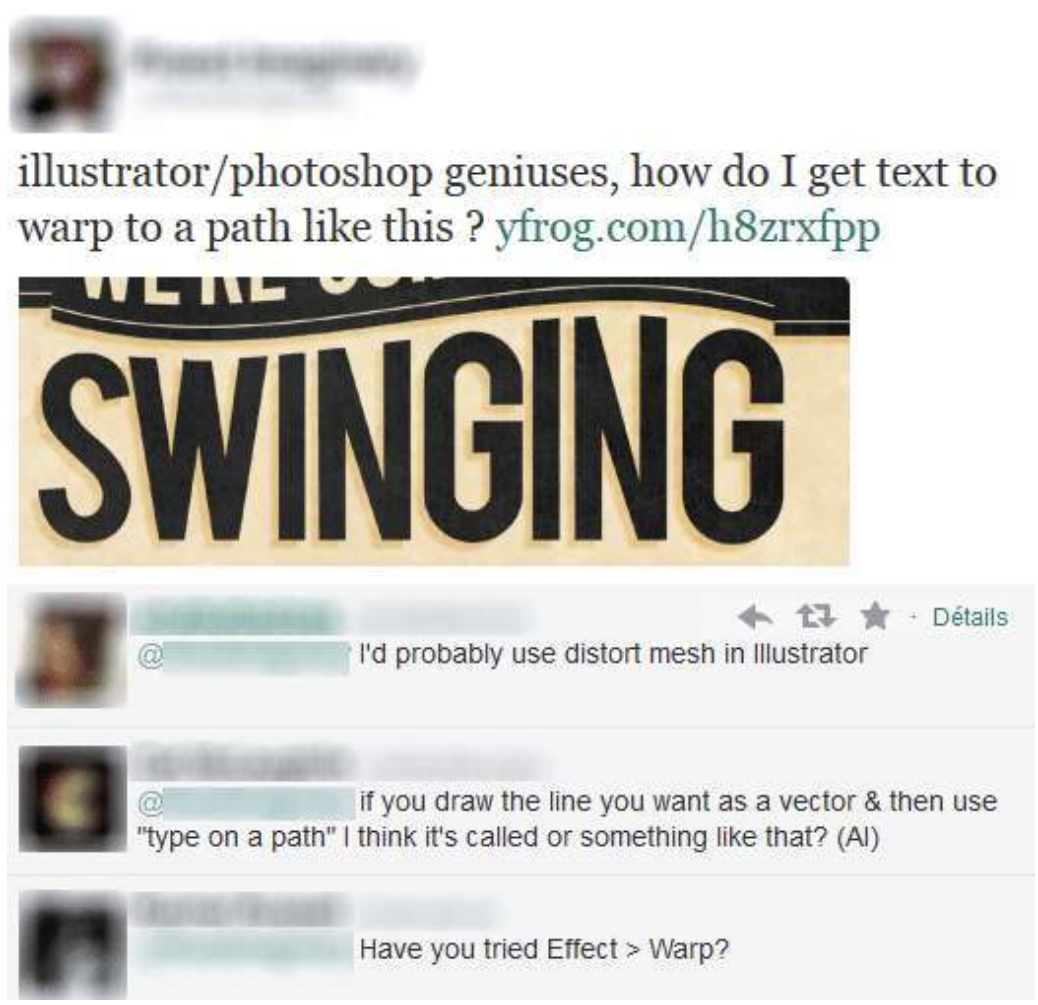

Bien que le recours à la foule permette d'obtenir rapidement des réponses factuelles de ce type, nous souhaitons mettre l'emphase ici sur un type de question en particulier, auquel on ne peut pas nécessairement répondre en invoquant des connaissances formelles, établies et consensuelles. Evans et al. (2010) ont d'ailleurs souligné la complémentarité des médias sociaux par rapport aux moteurs de recherche traditionnels, ces derniers étant mieux adaptés à la recherche des faits bruts, tandis que les premiers peuvent être mobilisés pour des questions faisant appel au «bon sens». En effet, nous avons vu plusieurs exemples de réponses destinées à partager des " pré-connaissances ${ }^{6}$, c'est-àdire des notions en développement, parfois conflictuelles, qui s'expriment sous la forme de nouvelles interrogations et de tentatives de réponses. Contrairement à Wikipedia, dont l'objectif est de répertorier l'ensemble des connaissances existantes, qui sont éditées, corrigées et validées par les internautes afin de produire une recension consensuelle, Twitter laisse place à la diffusion de "connaissances en devenir », par le biais de questions qui demeurent ouvertes et à des réponses qui demeurent incomplètes. Dans l'exemple illustré (cf. figure 6), un professeur s'interroge sur les enjeux liés à l'évaluation de la contribution asymétrique en situation de travail collaboratif. Ce questionnement appelle à une discussion autour d'expériences et d'hypothèses diverses. 


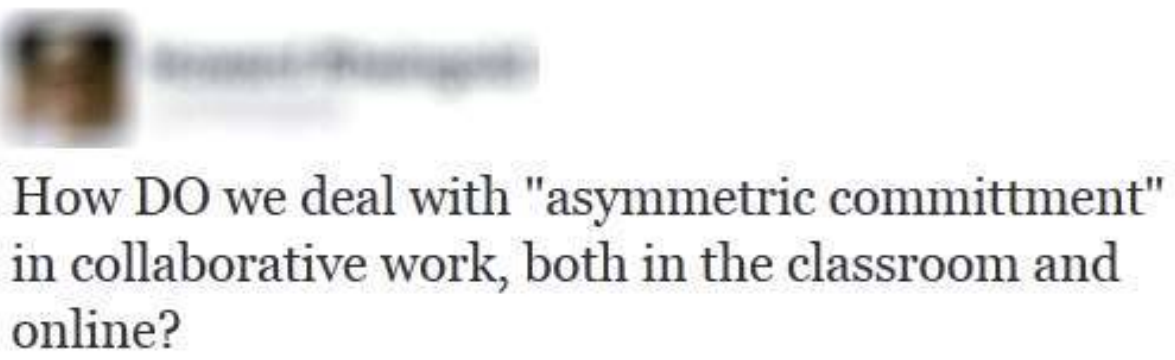

41 Ainsi, certains usagers de cette plateforme de microblogage laissent librement cours à l'expression de questionnements et d'hypothèses afin de voir ce que d'autres usagers en pensent. Ne serait-ce que par le nombre de réponses ou de commentaires reçus, ils peuvent évaluer l'intérêt de leur question et en retour voir si celle-ci vaut la peine d'être investiguée davantage. Ici, le fait de poser de bonnes questions peut avoir autant d'impact que d'y répondre.

\section{Vers une conceptualisation des affordances collaboratives de Twitter}

D'un point de vue théorique, les chercheurs s'inscrivant dans une perspective de la pratique (Corradi, Gherardi et Verzelloni, 2008) - et plus particulièrement ceux qui adoptent les approches de la sociomatérialité - envisagent les pratiques de travail en tant que configurations sociomatérielles devant être étudiées comme telles (Orlikowski et Scott, 2008) et considèrent que les changements organisationnels et technologiques sont mutuellement constitutifs et inter-reliés (Leonardi, 2009). En ce sens, le concept d'affordance (Gibson, 1979) a été mobilisé par Leonardi pour décrire les contraintes et possibilités inhérentes aux propriétés matérielles des outils numériques dans les organisations (Leonardi, 2010). Treem et Leonardi (2012) proposent d'ailleurs de développer une perspective "relationnelle " des affordances pour décrire la relation entre organisation et médias sociaux. Celle-ci permet de ne pas se borner à décrire seulement les fonctionnalités des outils et les usages qu'elles permettent pour se tourner vers les actes communicationnels rendus possibles par les médias sociaux. L'application du concept d'affordance avait d'ailleurs été habilement illustrée par Zammuto et al. (2007) qui ont traité de l'influence de la technologie sur les processus organisationnels de façon non-déterministe, en montrant comment certains agencements sociomatériels offrent de nouvelles possibilités « d'actions organisantes ».

Dans le même ordre d'idée, nous croyons que les usages informels que nous avons décrits tirent profit des spécificités matérielles et communicationnelles de la plateforme de microblogage étudiée et favorisent la mise en lumière de ses affordances collaboratives. Nous souhaitons ici apporter notre modeste contribution au développement du concept d'affordance en soulignant plus particulièrement les potentialités collaboratives de Twitter. Le tableau qui suit reprend les exemples relatés dans cet article pour permettre ultérieurement le développement d'une réflexion sur les liens entre ces différentes dimensions et propose quelques hypothèses relatives aux conséquences organisationnelles possibles. ${ }^{7}$ 
Tableau 1 : Tableau synthèse des usages identifiés et des affordances collaboratives qu'ils suggèrent

\begin{tabular}{|c|c|c|c|}
\hline $\begin{array}{l}\text { Usages } \\
\text { observés }\end{array}$ & $\begin{array}{l}\text { Caractéristiques } \\
\text { matérielles et } \\
\text { communication- } \\
\text { nelles de Twitter }\end{array}$ & Affordances collaboratives & $\begin{array}{l}\text { Conséquences } \\
\text { organisationnelles } \\
\text { possibles }\end{array}$ \\
\hline $\begin{array}{lr}\text { Raconter } & \text { le } \\
\text { travail en } \\
\text { train de } \\
\text { faire (working } \\
\text { out loud) }\end{array}$ & $\begin{array}{ll}\text { - Concision } & \text { des } \\
\text { messages } & \\
\text {-Diffusion } & \\
\text { publique } & \\
\text { défaut; } & \\
\text { - Mobilité. } & \end{array}$ & $\begin{array}{l}\text { Permet de créer un backchannel } \\
\text { conversationnel. }\end{array}$ & $\begin{array}{l}\text { Facilite la naissance de } \\
\text { réseaux collaboratifs } \\
\text { informels excédant les } \\
\text { frontières de } \\
\text { l'organisation. }\end{array}$ \\
\hline $\begin{array}{l}\text { Partager des } \\
\text { traces de son } \\
\text { travail }\end{array}$ & 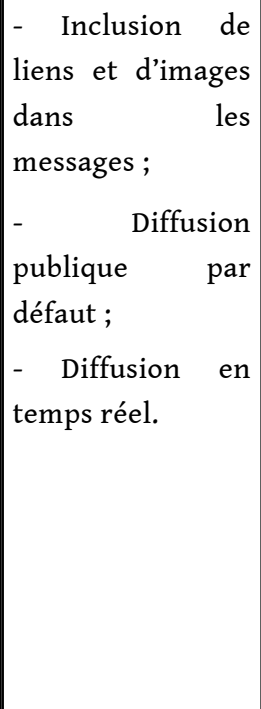 & $\begin{array}{l}\text { Permet les contributions } \\
\text { spontanées et itératives d'un public } \\
\text { plus large. }\end{array}$ & $\begin{array}{l}\text { - Impacts liés à la } \\
\text { diffusion d'une } \\
\text { production } \\
\text { " imparfaite " sur } \\
\text { l'image de marque de } \\
\text { l'entreprise; } \\
\text { - Risques de fuite } \\
\text { d'information } \\
\text { confidentielle; } \\
\text { - Le travail ouvert se } \\
\text { construit par des } \\
\text { "ajustements au fur } \\
\text { et à mesure " plutôt } \\
\text { que selon une } \\
\text { planification. }\end{array}$ \\
\hline $\begin{array}{l}\text { Diffuser ses } \\
\text { intentions }\end{array}$ & $\begin{array}{l}\text { - Diffusion } \\
\text { publique par } \\
\text { défaut; } \\
\text { - Diffusion en } \\
\text { temps réel. }\end{array}$ & $\begin{array}{l}\text { Permet d'évaluer l'intérêt d'une } \\
\text { idée et de repérer de potentiels } \\
\text { collaborateurs. }\end{array}$ & $\begin{array}{ll}\text { - Potentiel pour de } \\
\text { nouveaux modes de } \\
\text { coordination } & \text { de } \\
\text { l'action. } & \end{array}$ \\
\hline $\begin{array}{l}\text { Questionner } \\
\text { la foule }\end{array}$ & $\begin{array}{lr}\text { - Diffusion } \\
\text { publique par } \\
\text { défaut; } \\
\text { - Diffusion en } \\
\text { temps réel. } \\
\text { - Rediffusion (r } \\
\text { etweet). }\end{array}$ & $\begin{array}{l}\text { Permet de formuler collectivement } \\
\text { des réponses différentes de celles } \\
\text { fournies par les moteurs de } \\
\text { recherche (pré-connaissances, } \\
\text { hypothèses) }\end{array}$ & $\begin{array}{l}\text {-Potentiel pour de } \\
\text { nouveaux modes de } \\
\text { gestion et de partage } \\
\text { des connaissances. }\end{array}$ \\
\hline
\end{tabular}




\section{Conclusion}

44

Cette analyse a permis d'esquisser les contours d'un espace particulier de narration, de conversation et de collaboration qui est de plus en plus mobilisé par des travailleurs oeuvrant dans différents secteurs professionnels exigeant l'utilisation massive de ressources informationnelles. Alors que Twitter n'a pas été conçu à l'origine en tant que plateforme de conversation ni de collaboration, cet outil «public» de microblogage s'immisce de façon informelle dans leur quotidien.

Les exemples qui ont été présentés ont permis de jeter un premier coup d'œil sur la place qui leur est faite spontanément par les employés ayant volontairement décidé de les intégrer à leurs pratiques professionnelles. Ces exemples peuvent être catégorisés en quatre types d'usage : les récits portant sur le travail, l'ouverture et le partage de traces de son travail, la diffusion d'intentions et les questions à la foule. Notons que ces usages ont été observés chez une catégorie particulière de travailleurs, soit les knowledge workers. Rappelons que ceux-ci sont particulièrement habiles pour constituer des structures organisationnelles alternatives leur permettant d'étendre leur réseau (Payne, 2008). À cet effet, la plateforme de microblogage publique confère certains avantages par rapport aux médias sociaux d'entreprise fermés. Notamment, cela permet aux travailleurs du savoir de disposer d'emblée de la masse critique d'usagers qui fait souvent défaut lorsqu'un outil d'entreprise repose sur la participation des employés seulement. Pour ces travailleurs, qui sont constamment en contact avec des personnes à l'extérieur de leur organisation, il est plus efficace de recourir à ce type de plateforme que de diviser son attention et ses efforts à des flux informationnels organisés en silos (collègues, partenaires externes, clients, amis, famille, etc.).

Alors que les organisations investissent beaucoup de temps, de ressources humaines et financières dans l'implantation et la maintenance d'outils de communication et de collaboration sophistiqués (messagerie interne, visioconférence, collecticiel, etc.), pourquoi les travailleurs ressentent-ils le besoin d'utiliser des outils autres que ceux prescrits par leur organisation de travail ? Quelles fonctions collaboratives particulières retrouvent-ils au sein d'une plateforme de microblogage externe en comparaison à celles qui sont formellement supportées et prescrites par leur employeur?

Ces questions devront faire l'objet d'un examen particulier. Il serait pertinent, nous semble-t-il, d'interroger les usagers sur leurs motivations et les bénéfices qu'ils en retirent. Pour le moment, seules quelques hypothèses peuvent être formulées en fonction des types d'usages que nous avons observés. La transposition, par exemple, dans le milieu de travail de pratiques déjà ancrées dans leur vie personnelle - avec un outil déjà bien maîtrisé - semble aller de soi pour les travailleurs, qui n'ont pas ainsi à investir d'efforts supplémentaire dans l'apprentissage et l'intégration d'un nouvel outil de travail. À ce sujet, il n'est pas étonnant de constater que de plus en plus d'organisations commencent à autoriser leurs employés à utiliser leurs propres appareils au travail. Cette tendance, désignée par l'expression «Bring your Own Device » (BYOD) a d'ailleurs été remarquée par l'entreprise Cisco. Une étude réalisée par cette dernière auprès de sociétés américaines a révélé que $95 \%$ des organisations sondées autorisent leurs employés à utiliser leurs appareils personnels au travail et que 36\% d'entre elles allaient jusqu'à en effectuer le support (Cisco, 2012). Ces données préliminaires suggèrent que les 
employeurs se font progressivement à l'idée de laisser leurs employés choisir leurs propres outils plutôt que de leur en imposer d'autres.

En somme, le coût cognitif lié à l'utilisation de Twitter semble moins élevé que d'autres outils d'entreprise plus sophistiqués. À partir du moment où l'on reconnaît que ce type de plateforme permet aux travailleurs d'accéder à des informations auxquelles ils n'auraient pas accès à l'interne, cela nous oblige aussi à nous interroger sur le devenir des travailleurs qui n'y ont pas accès. Seront-ils désavantagés dans leur capacité d'obtenir et de partager l'information dont ils ont besoin pour effectuer leur travail?

$\mathrm{Au}$ fur et à mesure que les usages informels du microblogage au travail s'intensifient, il est probable qu'ils occasionnent certaines perturbations dans les pratiques institutionnalisées existantes. Si cela se vérifiait, il deviendrait important d'étudier comment les organisations font face à ces situations et d'évaluer de quelle manière ces initiatives " par le bas " peuvent trouver leur place au sein des processus formels de l'organisation. Et dans le cas où ces nouveaux usages collaboratifs n'ont pas pu trouver leur place au sein des pratiques institutionnalisées, reste qu'ils font appel à l'organisation même du travail et à la gestion des connaissances qui y est associée. À ce propos, une question se pose : comment les organisations de travail gèrent-elles l'utilisation d'outils qu'elles ne contrôlent pas totalement et qui permettent un accès public à du contenu potentiellement sensible ou même confidentiel? Lorsque les travailleurs se constituent des réseaux en ligne pour partager les connaissances, au-delà des frontières formelles de l'organisation, cela pose nécessairement de nombreuses questions en termes de qualité de l'information, de confiance, d'intégration ou encore en matière de pérennité des connaissances et de frontière entre vie privée et vie professionnelle, entre autres. C'est pourquoi Il apparaît nécessaire de poursuivre des investigations qui permettront de dégager les différents enjeux organisationnels liés à ces pratiques.

\section{BIBLIOGRAPHIE}

Bailey, D. E., P. M. Leonardi et J. Chong. 2009. «Minding the Gaps: Understanding Technology Interdependence and Coordination in Knowledge Work». Organization Science, vol. 21, no 3, p. 713-730.

Bødker, S., et E. Christiansen. 2006. «Computer Support for Social Awareness in Flexible Work». Computer Supported Cooperative Work, vol. 15, no 1, p. 1-28.

Bonneau, C. 2012. «Joindre une conversation sans y avoir été invité: les normes éthiques informelles des acteurs commerciaux pour l'envoi de réponses non-sollicitées sur Twitter». In Médias sociaux: enjeux pour la communication, S. Proulx, M. Millette et L. Heaton, p. 247-257. Québec: Presses de l'Université du Québec.

Bonneau, C., et P.-L. Harvey. 2006. Scénarios transactionnels sur internet: l'application de la méthode du coût généralisé:Proceedings of the 18th international conference on Association Francophone d'Interaction Homme-Machine (Montréal, Canada). ACM Press, p. 91-98 
Cardon, D. 2008. «Le design de la visibilité : essai de cartographie du Web 2.0». Réseaux, no 152, p. 93-137.

Cisco (2012). BYOD and Virtualization: Insights from the Cisco IBSG Horizons Study En ligne. < http://www.slideshare.net/CiscoIBSG/byod-and-virtualization-insights-from-the-cisco-ibsghorizons-study>. Consulté le 30 mai 2012.

Corradi, G., S. Gherardi et L. Verzelloni. 2008. Ten good reasons for assuming a 'practice lens' in organization studies:OLKC Conference (University of Aarhus, Copenhague, 28-30 avril).

Crague, G. 2003. «Des lieux de travail de plus en plus variables et temporaires». Economie et statistique, vol. 369, no 1, p. 191-212.

Croft, A. C. 2007. «Emergence of "New" Media Moves PR Agencies in New Directions». Public Relations Quarterly, vol. 52, no 1, p. 16-20.

Dann, S. 2010. «Twitter content classification». First Monday, vol. 15, no 12.

Diffley, S., J. Kearns, W. Bennett et P. Kawalek. 2011. «Consumer Behaviour in Social Networking Sites: Implications for Marketers». Irish Journal of Management, vol. 30, no 2, p. 47-66.

Diga, M., et T. Kelleher. 2009. «Social media use, perceptions of decision-making power, and public relations roles». Public Relations Review, vol. 35, no 4, p. 440-442.

DiMicco, J., D.R. Millen, W. Geyer, C. Dugan, B. Brownholtz et M. Muller. 2008. Motivations for social networking at work:Proceedings of the 2008 Conference on Computer Supported Cooperative Work (New York). ACM, p. 711-720

Domenget, J.-C. 2012. «De l'impératif de visibilité aux enjeux éthiques: les usages de Twitter par des professionnels du Web». In Médias sociaux: enjeux pour la communication, S. Proulx, M. Millette et L. Heaton, p. 217-232. Québec: Presses de l'Université du Québec.

Ehrlich, K., et N.S. Shami. 2010. Microblogging inside and outside the workplace:Proceedings of the 4th International AAAI Conference on Weblogs and Social Media (ICWSM 2010) (Menlo Park, CA). p. 42-49

Evans, B. M., S. Kairam et P. Pirolli. 2010. «Do your friends make you smarter?: An analysis of social strategies in online information seeking». Information Processing \& Management, vol. 46, no 6, p. 679-692.

Faraj, S., et S. L. Johnson. 2010. «Network Exchange Patterns in Online Communities». Organization Science, vol. 22, no 6, p. 1464-1480.

Gibson, J. J. 1979. The Ecological Approach to Visual Perception. Boston: Houghton Mifflin p. Granovetter, M.S. 1973. «The strength of weak ties». American journal of sociology, vol. 78, no 6, p. 1360-1380.

Hasan, H., et C. C. Pfaff. 2006. The Wiki: an environment to revolutionise employees' interaction with corporate knowledge:OZCHI 2006 (2006). p. 377-380

Hine, C. 2000. Virtual ethnography. Londres: Sage, 192 p.

Honeycutt, C., et S.C. Herring. 2009. Beyond microblogging: Conversation and collaboration via Twitter: Proceedings of the 42nd International Conference on System Sciences (Hawaii). IEEE, p. 1-10

Huberman, B.A., D.M. Romero et F. Wu. 2009. «Social networks that matter: Twitter under the microscope». First Monday, vol. 14, no 1.

Huh, J., A. Arbor, J. Thomas, L. Jones, Y. Heights, T. Erickson, W. Kellogg et R. Bellamy. 2007. BlogCentral: The Role of Internal Blogs at Work:CHI 2007 Proceedings (2007). p. 2447-2452 
Jackson, A., J. Yates et W.J. Orlikowski. 2007. «Corporate Blogging: Building community through persistent digital talk». 2007 40th Annual Hawaii International Conference on System Sciences.

Ladner, S. 2008. «Laptops in the Living Room: Mobile Technologies and the Divide between Work and Private Time among Interactive Agency Workers». Canadian Journal of Communication, vol. 33, no 3.

Leonardi, P. M. 2009. «Crossing the Implementation Line: The Mutual Constitution of Technology and Organizing Across Development and Use Activities». Communication Theory, vol. 19, no 3, p. 278-310.

Leonardi, P.M. 2010. «Digital materiality? How artifacts without matter, matter». First Monday, vol. 15 , no 6.

Liao, Q., S. Pan, J. C. Lai et C. Yang. 2011. Enterprise Blogging in a Global Context : Comparing Chinese and American Practices:CSCW 11 (2011). p. 35-44

Licoppe, C., R. Cudicio et S. Proulx. 2011. «"Présence connectée" au travail : les usages de la messagerie instantanée, le genre des "questions rapides" et l'économie morale de la "contribution"». ethnographiques.org, no 23.

Lorenzo-Romero, C., E. Constantinides et M. Alarcón-del-Amo. 2011. «Consumer adoption of social networking sites: implications for theory and practice». Journal of Research in Interactive Marketing, vol. 5, no 2/3, p. 170-188.

McAfee, A.P. 2006. «Enterprise 2.0: The dawn of emergent collaboration». MIT Sloan Management Review, vol. 47, no 3, p. 21-28.

Miles, M.B., et A.M. Huberman. 1994. Qualitative data analysis: An expanded sourcebook: Sage Publications, $336 \mathrm{p}$.

Nardi, B.A., S. Whittaker et H. Schwarz. 2000. «It's not what you know it's who you know». First Monday, vol. 5, no 5.

Nichols, J., et J.-H. Kang. 2012. Asking Questions of Targeted Strangers on Social Networks:CSCW 12 (2012). p. 999-1002

Office québécois de la langue française. 2011. «Mot-clic». Grand dictionnaire terminologique. En ligne. <http://www.granddictionnaire.com >. Consulté le 24 avril 2012.

Orlikowski, W.J., et S.V. Scott. 2008. «Sociomateriality: Challenging the Separation of Technology, Work and Organization». The academy of management annals, vol. 2, no 1, p. 433-474.

Papacharissi, Z. 2009. «The virtual geographies of social networks: a comparative analysis of Facebook, LinkedIn and ASmallWorld». New Media \& Society, vol. 11, no 1-2, p. 199-220.

Paquet, S. 2011. «Why Quora Is Not Wikipedia». The Quora Review. En ligne. <http:// quorareview.com/2011/02/03/why-quora-is-not-wikipedia/>. Consulté le 26 avril 2012.

Payne, J. 2008. «Using wikis and blogs to improve collaboration and knowledge sharing». Strategic HR Review, vol. 7, no 3, p. 5-12.

Pew Internet \& American Life Project. 2011. «Twitter Update 2011». Pew Research Center Publications. En ligne. <http://pewresearch.org/pubs/2007/twitter-users-cell-phone-2011demographics>. Consulté le 24 avril 2012.

Proulx, S., M. Millette et L. Heaton (2012). Médias sociaux: enjeux pour la communication, Presses de l'Université du Québec. 
Robert Half Technologies. 2011. «Social Work? More Companies Permit Social Networking on the Job, Robert Half Technology Survey Reveals». Newswire.ca. En ligne. <http://www.newswire.ca/ $\mathrm{fr} /$ story/757345/social-work-more-companies-permit-social-networking-on-the-job-robert-halftechnology-survey-reveals>. Consulté le 23 janvier 2011.

Rooksby, J., et I. Sommerville. 2011. «The Management and Use of Social Network Sites in a Government Department». Computer Supported Cooperative Work (CSCW), p. 397-415.

Rybalko, S., et T. Seltzer. 2010. «Dialogic communication in 140 characters or less: How Fortune 500 companies engage stakeholders using Twitter». Public Relations Review, vol. 36, no 4, p. 336-341.

Sakaki, T., M. Okazaki et Y. Matsuo. 2010. Earthquake shakes Twitter users: real-time event detection by social sensors:Proceedings of the 19th international conference on World wide web. ACM, p. 851-860

Steinfield, C., M. Huysman et P. Leonardi. 2012. «Special Issue Call for Papers: Social Media and Communication in the Workplace». Journal of Computer-Mediated Communication. En ligne. <http:// tc.msu.edu/jcmc_cfp_social_media>. Consulté le 23 janvier 2012.

Stocker, A., A. Richter, P. Hoefler et K. Tochtermann. 2012. «Exploring Appropriation of Enterprise Wikis». Computer Supported Cooperative Work (CSCW), vol. 21, no 2-3, p. 317-356.

Tay, G. 2011. «Ask Five Questions to Determine Whether to Deploy Social Software Bottom-Up or Top-Down». Gartner Research.

Treem, J. W., et P. M. Leonardi. 2012. «Social Media Use in Organizations: Exploring the Affordances of Visibility, Editability, Persistence, and Association». Communication Yearbook, vol. 36, p. 143-189.

Twitter. 2012. «Twitter turns six». Twitter blog. En ligne. <http://blog.twitter.com/2012/03/ twitter-turns-six.html>. Consulté le 24 avril 2012.

von Kaufmann, R. 2009. «Intention Broadcasting: A Model for Computer-Mediated Intention Sharing and Coordination». Proceedings of I-KNOW' 09 and I-SEMANTICS '09.

Wajcman, J., et E. Rose. 2011. «Constant Connectivity: Rethinking Interruptions at Work». Organization Studies, vol. 32, no 7, p. 941-961.

Williams, B. 2010. «When will we Work Out Loud? Soon!». TheBrycesWrite. En ligne. <http:// thebryceswrite.wordpress.com/2010/11/29/when-will-we-work-out-loud-soon/>.

Winer, D. 2009. «Narrate Your Work». Scripting News. En ligne. <http://scripting.com/ stories/2009/08/09/narrateYourWork.html>. Consulté le 1 avril 2012.

Yardi, S., S. A. Golder, U. Hall et M. J. Brzozowski. 2009. Blogging at Work and the Corporate Attention Economy:CHI 09 (2009). p. 1-10

Zammuto, R.F., T.L. Griffith, A. Majchrzak, D.J. Dougherty et S. Faraj. 2007. «Information technology and the changing fabric of organization». Organization Science, vol. 18, no 5, p. 749-762.

Zhang, J., Y. Qu, J. Cody et Y. Wu. 2010. A case study of micro-blogging in the enterprise: use, value, and related issues:Proceedings of the 28th international conference on Human factors in computing systems (Atlanta, Georgia, USA, 2010). ACM, p. 123-132

Zhao, D., et M.B. Rosson. 2009. How and why people Twitter: the role that micro-blogging plays in informal communication at work:Proceedings of the ACM 2009 international conference on Supporting group work (Sanibel Island, Florida). ACM, p. 243-252 


\section{NOTES}

1. Nous remercions Renaud Carbasse pour son aide précieuse dans la réalisation de cette revue de littérature.

2. Traduction libre de l'expression marker of addressivity utilisée par Honeycutt et Herring (2009).

3. Le terme mot-clic, formé à partir de mot-clé et de clic, a été proposé par l'office québécois de la langue française (2011) en guise de traduction de hashtag.

4. Bien que leur compte soit public, nous avons camouflé leurs noms d'usager ou toute autre marque permettant l'identification pour préserver leur anonymat.

5. Dave Winer est un pionnier du Web. On lui attribue, entre autres, le développement des premiers outils de blogues, de syndication de contenu (RSS), de podcasting et de logiciels de gestion de contenu. Contributeur au magazine Wired, il a aussi été chercheur à la Harvard Law School, en plus d'être un entrepreneur et d'avoir investi dans plusieurs compagnies associées aux nouveaux médias.

6. Nous empruntons le terme "pré-connaissance" (pre-knowledge) à Seb Paquet (2011) qui l'utilise pour caractériser le contenu du site Quora, une plateforme web disponible au public depuis 2010 qui permet à ses utilisateurs de créer, d'éditer et d'organiser des questions-réponses. 7. Les prochaines étapes de notre recherche seront consacrées à l'exploration et au raffinement de ces pistes et nous espérons que d'autres chercheurs puissent les enrichir à partir de leurs travaux respectifs.

\section{RÉSUMÉS}

Bien que les organisations soient de plus en plus nombreuses à implanter des réseaux sociaux d'entreprise destinés en interne à être utilisés par leurs employés, les conversations portant sur le travail empruntent aussi d'autres réseaux : les médias sociaux numériques (rsn) «publics». Ainsi, il devient important de prendre en compte les pratiques collaboratives au travail selon les échelles et modalités nouvelles offertes par des plateformes qui traversent les sphères d'activités privées et publiques des travailleurs. La plateforme de microblogage Twitter est utilisée notamment par certains travailleurs pour diffuser des connaissances tacites ou formalisées, mais aussi les hypothèses et pistes de réflexion qui jalonnent leurs pratiques professionnelles. Alors que Twitter n'a pas été conçu à l'origine pour la conversation ni la collaboration, cette plateforme de microblogage s'immisce de façon informelle dans le quotidien des travailleurs, en leur permettant de communiquer en temps réel, via un canal public qui n'est pas nécessairement autorisé, reconnu ni contrôlé par leur organisation de travail, avec un public excédant les frontières de l'entreprise. Ainsi, les commentaires et les interactions que suscitent ces diffusions peuvent favoriser l'émergence de réseaux informels de collaboration. Cet article tente d'identifier et de caractériser les différentes formes de pratiques informelles de mise en visibilité du travail et de collaboration numérique par l'entremise de la plateforme de microblogage Twitter. Les exemples présentés permettent d'illustrer la place faite spontanément à cette plateforme par les travailleurs ayant volontairement décidé de l'intégrer dans leurs pratiques professionnelles. Nous distinguons quatre types d'usages : les récits portant sur le travail, 
l'ouverture et le partage de traces de son travail, la diffusion d'intentions et les « questions à la foule ».

Nowadays, a growing number of organizations implement corporate social network tools intended for internal use. However, work-related conversations also circulate on "public" (i.e. external) social media sites. Scholars studying collaborative practices in the workplace need to take this public channel in consideration, even if it is neither recognized, authorized nor controlled by the employer. Twitter, for example, is a microblogging site used by some workers to share not only their tacit or formal knowledge, but also to make hypotheses, intentions, reflections, problems and questions visible in real time outside the formal boundaries of the organization. Even though Twitter was not originally created as a conversational or collaboration platform, we observe different form of informal collaboration practices involving the use of this tool by employees who have decided to integrate it in their professional lives. This article presents empirical examples illustrating the following types of uses: "working out loud" narratives, sharing traces of "work-in-progress," broadcasting intentions and asking questions of the crowd.

Si bien cada vez son más numerosas las organizaciones que optan por implantar redes sociales empresariales destinadas al uso interno, por parte de sus empleados, las conversaciones sobre temas relacionados con el trabajo pueden realizarse a través de otras redes: los medios sociales digitales «públicos». Por tanto, parece indispensable analizar las prácticas colaborativas en el ámbito del trabajo, en función de las nuevas escalas y de las nuevas modalidades que ofrecen aquellas plataformas que atraviesan las esferas de las actividades privadas y públicas de los empleados. Así, además de considerar la plataforma de microblogging Twitter, que es especialmente utilizada por algunos trabajadores con el propósito de difundir conocimientos tácitos o formales, se analizan también las hipótesis y los puntos de reflexión que están en la base de sus prácticas laborales. Si bien Twitter no ha sido concebida en sus orígenes como una plataforma de conversación ni de colaboración, esta herramienta de microblogging se inmiscuye de manera informal, en el día a día de los trabajadores, permitiéndoles comunicarse, en tiempo real, con un público que excede las fronteras de la organización en la que trabajan, a través de un canal público que no necesariamente ha sido autorizado, reconocido ni controlado por aquella. De esta forma, los comentarios y las interacciones que suscitan estas publicaciones pueden dar lugar al nacimiento de redes informales de colaboración. En el presente artículo se identifican y se caracterizan las distintas modalidades de prácticas informales, sobre el trabajo, y de colaboración digital a través de Twitter. Los ejemplos que aquí se presentan permiten ilustrar el lugar que, espontáneamente, han concedido a Twitter los empleados que voluntariamente han decidido integrarla en sus prácticas profesionales. En este sentido, se distinguen cuatro tipos de usos: los relativos al ámbito laboral, la apertura y transmisión de aspectos de su trabajo, la difusión de intenciones y las preguntas dirigidas a las masas.

\section{INDEX}

Mots-clés : Microblogage, Twitter, collaboration, travail, pratiques informelles.

Keywords : Microblogging, work, informal practices.

Palabras claves : colaboración, trabajo, prácticas informales 


\section{AUTEUR}

\section{CLAUDINE BONNEAU}

Claudine Bonneau est professeure au Département de management et technologie à l'École des sciences de la gestion de l'UQAM. Elle détient un doctorat en communication de l'UQAM. Ses travaux de recherche s'inscrivent dans les courants de l'informatique sociale, du travail coopératif assisté par l'ordinateur ( $\mathrm{CSCW}$ ) et des études organisationnelles. Elle s'intéresse aux usages des médias sociaux, des technologies collaboratives et des logiciels libres à des fins professionnelles et pédagogiques. 